incomplete job histories, crudeness of exposure assessment, latent confounding, and survivor effect.

Conclusions We found evidence of an association between three two-digit occupation groups and incident stroke. We will better contextualize these results by refining the exposure assessment by examining associations with more detailed three-digit occupation groups, include all jobs held prior to enrollment, and incorporate employment duration.

\section{0-140 OCCUPATIONAL NOISE EXPOSURE AND METABOLIC SYNDROME}

Zara Ann Stokholm, Knet Lodberg Christensen, Jesper Medom Vestergaard, Åse Marie Hansen, Jens Peter Ellekilde Bonde, Henrik Albert Kolstad.

\subsection{6/OEM-2021-EPI.23}

Objective Metabolic syndrome, a major risk factor for cardiovascular disease and diabetes, is recognized as an important health problem. Both environmental and occupational noise exposure has been related to increased risk of cardiovascular disease. We examined if metabolic syndrome was associated to occupational noise exposure.

Methods Associations between quantitative measures of occupational noise exposure and metabolic syndrome were analysed with logistic regression in 411 industrial- and 154 finance and service workers selected as a random sample from 12 industries between 2009 and 2010. We used anthropometric measures and biomarkers to define metabolic syndrome as abdominal obesity and the presence of $\geq 2$ of the following conditions: high blood pressure, high triglycerides, low HDL cholesterol and high HbA1c.

Results A total of 167 workers were classified with metabolic syndrome. After adjustment for potential confounders, prevalence ratios for metabolic syndrome comparing the highest to the lowest noise exposure quartiles were 2.04 (95\% CI: 1.034.03) for cumulative occupational noise exposure. In stratified analyses, the association diminished for industrial workers and increased for finance and service workers.

Conclusion These cross-sectional findings suggest cumulative occupational noise exposure may contribute to cardiometabolic health, but results need to be confirmed with prospective data.

\section{0-144 RISK OF MYOCARDIAL INFARCTION AMONG PIGEON BREEDERS EXPOSED TO ORGANIC DUST}

${ }^{1}$ Sandra lleby Rokkedrejer, Vivi Schlünssen, Henrik Albert Kolstad, Jesper Medom Vestergaard, Christine Cramer, Martin Byskov Kinnerup. 'Aarhus University Hospital, Denmark

\subsection{6/OEM-2021-EPI.24}

Introduction Anthropogenic airborne particulate matter is associated with ischemic heart disease, while little is known about particles from organic dust.

Objective Pigeon breeders are exposed to high levels of organic dust in the pigeon lofts, and the objective of this study is to investigate the association with myocardial infarction.

Methods We followed 6,256 male pigeon breeders and their 1:30 individually matched referents from 1980 or first year of membership in the Danish Racing Pigeon Association if later, until end of study in 2013. Referents were matched on sex and year of birth and randomly drawn from the general Danish population. Information on hospital-based diagnoses, emigration, death and confounders were obtained by record linkage with Danish national registers. Subjects with a diagnosis of myocardial infarction, or chronic ischemic heart disease prior to start of follow-up were excluded. Stratified Cox regression analyses estimated the hazard ratios (HR) of myocardial infarction, adjusted for occupation and place of residence (urban/rural) at start of follow-up.

Results The incidence rate of myocardial infarction was 507 (per 100,000 person-years) among pigeon breeders and 445 among the referents. The crude hazard ratio was $1.16 \quad(95 \%$ CI,1.06-1.26), similar after adjusting for possible confounding variables; 1.12 (95\% CI,1.03-1.23).

Conclusion In this study we found an increased risk of myocardial infarction among male pigeon breeders. The excess risk is suggested to be explained by exposure to organic dust, pointing to organic dust being a part of ischemic heart disease aetiology. We partly adjusted for lifestyle factors, but the lack of individual information on ex. smoking and dietary factors is a clear limitation. Thus, findings must be interpreted with caution even if adjustment by occupation and place of residence may have reduced such potential confounding. Future research with more detailed information on organic dust exposure and lifestyle factors is warranted.

\section{0-190 THE OCCUPATIONAL DISTRIBUTION OF METS PREVALENCE AND INCIDENCE DIFFERS BY SEX AND IS NOT EXPLAINED BY AGE AND HEALTH BEHAVIOR: RESULTS FROM 75,000 DUTCH WORKERS FROM 40 OCCUPATIONAL GROUPS}

'Sander van Zon, Benjamin C Amick, Sandra Brouwer, Ute Bültmann, Trynke R de Jong. ${ }^{1}$ University Medical Center Groningen, University of Groningen, Netherlands

\subsection{6/OEM-2021-EPI.25}

Objective This study examines the association between 40 occupational groups and baseline prevalence and incidence of metabolic syndrome (MetS), separately for male and female workers, and whether age and health behaviors can explain the association.

Methods Data from 74,857 Lifelines Cohort and Biobank Study participants were used to regress occupational group membership, coded by Statistics Netherlands, on the prevalence and incidence of MetS using Logistic and Cox-regression analyses. MetS diagnosis was based on physical examinations, blood analysis, and recorded medication use. Information on age, smoking status, physical activity, diet and alcohol consumption was acquired using questionnaires.

Results MetS prevalence was $17.5 \%$ for males and $10.6 \%$ for females. In the fully adjusted models, three occupational groups were associated with increased MetS prevalence in both sexes. Three additional occupational groups were associated with MetS among men, nine among women. Strongest associations were found for male 'hospitality, retail and other service managers' (odds ratio (OR): 1.65; 95\% confidence interval (CI): 1.03-2.65) and female 'stationary plant and machine operators' (OR: 3.44; 95\% CI: 1.57-4.54). During a median 3.8-year follow-up, MetS incidence was $7.8 \%$ for 